\title{
Phenotypic expression and prevalence of ESBL-producing Enterobacteriaceae in samples collected from patients in various wards of Mulago Hospital, Uganda
}

John N Kateregga*, Ronah Kantume, Collins Atuhaire, Musisi Nathan Lubowa and James G Ndukui

\begin{abstract}
Background: Resistance to extended-spectrum cephalosporins among Enterobacteriaceae has been reported yet they serve as the last line treatment for severe infections in Uganda and other countries. This resistance often leads to nosocomial infection outbreaks and therapeutic failures from multidrug resistant bacteria. The main objective of this study was to determine the prevalence of extended-spectrum beta-lactamase (ESBL)-producing Enterobacteriaceae in clinical samples of patients in various wards of Mulago Hospital; Uganda's main national referral and teaching hospital.

Methods: This cross-sectional study was conducted between January-April, 2014. Purposive consecutive sampling was used to collect pus swab, urine, blood and CSF samples from patients in the various wards. A total of 245 consecutive, non-repetitive, clinical samples were obtained and tested for phenotypic ESBL production using the Double Disc Synergy Test using cefotaxime, ceftazidime, cefotaxime-clavulanic acid and ceftazidime-clavulanic acid.

Results: Results show that $47 \%$ of the 245 samples had Enterobacteriaceae isolates. Of these isolates $62 \%$ were ESBL producers while $38 \%$ were of non-ESBL phenotype. E. coli was the most isolated organism (53.9\%), followed by $K$. pneumoniae (28.7\%). Majority of Enterobacteriaceae organisms were isolated from urine samples, followed by pus samples and of these $64.9 \%$ and $47.4 \%$ were ESBL-producers respectively. Klebsiella pneumoniae had the highest percentage of ESBL producers ( $72.7 \%$ ). There was a higher percentage of isolates showing resistance to ceftazidime (73\%) compared to cefotaxime $(57.5 \%)$. All Enterobacter cloacae isolates showed resistance to ceftazidime. There were no statistically significant association between phenotype (ESBL/non-ESBL) and patients' age or gender or Enterobacteriaceae spp.

Conclusions: This study reveals a high prevalence of ESBL producing organisms in Mulago Hospital and high levels of resistance to third generation cephalosporins. In addition to undertaking appropriate infection control measures, there is urgent need for formulation of an antibiotic policy in Uganda to prevent spread of these organisms. This also calls for continuous monitoring and reporting of the presence of such organisms in order to ensure rational and judicious use of antibiotics by clinicians.
\end{abstract}

Keywords: ESBL, Enterobacteriaceae, Cefotaxime, Ceftazidime, Clavulanic acid

\footnotetext{
* Correspondence: katereggaj@covab.mak.ac.ug

College of Veterinary Medicine, Animal Resources \& Biosecurity, Makerere University, P.O Box 7062, Kampala, Uganda
} 
Table 1 Criteria for determining the potency of the test antibiotics

\begin{tabular}{lll}
\hline ESBLS & E. coli ATCC 25922 & K. pneumoniae ATCC 700603 \\
\hline Ceftazidime $30 \mu \mathrm{g}$ & $(25-32 \mathrm{~mm})$ & $(22-29 \mathrm{~mm})$ \\
Cefotaxime $30 \mu \mathrm{g}$ & $(29-35 \mathrm{~mm})$ & $(18-22 \mathrm{~mm})$
\end{tabular}

The antibiotics had to show inhibition zone diameters in the above ranges in order to be used in the study

\section{Background}

Production of $\beta$-lactamase enzymes that hydrolyze the $\beta$-lactam ring is a predominant resistance mechanism for many Gram-negative bacteria including Enterobacteriaceae such as E. coli, Klebsiella pneumoniae, Klebsiella oxytoca, Pseudomonas aeruginosa, Proteus mirabilis, Enterobacter cloacae and Aeromonas spp. [1]. ExtendedSpectrum $\beta$-Lactamase (ESBL)-producing bacteria are capable of expressing these enzymes and this confers bacterial resistance to penicillins; first, second, and third-generation cephalosporins and aztreonam. ESBL-producing bacteria have been isolated in many parts of North America and Europe [2] and in Africa. In one South African hospital, 36 $\%$ of $K$. pneumoniae isolates were ESBL-producers and outbreaks of infections due to Klebsiella strains resistant to third-generation cephalosporins have also been reported in Nigeria and Kenya [3, 4]. In Uganda there has been lack of published information about ESBL-producers among organisms isolated from patients.

ESBL producers have a wide clinical significance and potential impact in healthcare settings especially in low income countries such as Uganda. The selection pressure and overuse of new antibiotics in the treatment of patients leads to selection for new variants of $\beta$-lactamase producers. ESBL producers are associated with various infections in virtually all body organs leading to meningitis, pneumonia, urinary tract infections, septicaemia and intra-abdominal infections $[5,6]$. Other conditions include osteomyelitis, endophthalmitis, pyomyositis and wound infections [7].

The possible spread of ESBL-producing organisms in a clinical setting is real. ESBL-associated antibiotic resistance causes increased morbidity and mortality; and hampers the control of infectious diseases. This in turn leads to increase in durations of illness and hospital stay; increase in health-care costs and more economic burden
Table 3 Enterobacteriaceae species isolated from patient samples

\begin{tabular}{lcc}
\hline Isolates & Frequency & Percent \\
\hline E. coli & 62 & 53.9 \\
Klebsiella pneumoniae & 33 & 28.7 \\
Proteus mirabilis & 16 & 13.9 \\
Enterobacter cloacae & 4 & 3.5 \\
Total & 115 & 100.0 \\
\hline
\end{tabular}

E. coli was the predominant isolate

to families. This study sought to determine the prevalence of ESBL-producing bacteria among isolates from samples in various wards of Mulago Hospital, the main national referral hospital in Uganda with a bed-capacity of 1,600 .

\section{Methods}

\section{Study design}

This was a cross-sectional study conducted between January-April 2014 to determine the prevalence of ESBLproducing Enterobacteriaceae in clinical samples collected from in-patient and out-patient wards of Mulago Hospital. The samples collected included urine, swabs (oral, HVS, wound), blood and CSF. Escherichia coli, Klebsiella pneumoniae, Proteus mirabilis and Enterobacter cloacae bacteria were isolated from the samples. The number of samples containing ESBL-producing Enterobacteriaceae was recorded. The prevalence of ESBL phenotypes among the isolates was determined.

\section{Sample size}

The sample size was determined using the formula advanced by Kish and Leslie [8]. Basing on results of a previous study in a similar setting, a prevalence of $20 \%$ [9] and confidence interval of $95 \%$ were used in the formula. The sample size was estimated to be about 245 samples.

\section{Sample collection and bacterial isolation}

Samples from 25 wards of Mulago Hospital were aseptically collected by purposive consecutive sampling from patients who gave informed written consent; properly

Table 2 Baseline characteristics of patients whose samples had Enterobacteriaceae isolates

\begin{tabular}{|c|c|c|c|c|}
\hline & $\mathrm{ESBL} n=71(61.7 \%)$ & Non-ESBL n= 44 (38.3\%) & Total & $\mathrm{p}$ value \\
\hline \multicolumn{5}{|l|}{ Sex } \\
\hline Female & 37 (52.1%) & $30(68.2 \%)$ & 67 (58.3\%) & 0.089 \\
\hline Male & $34(47.9 \%)$ & $14(31.8 \%)$ & $48(41.7 \%)$ & \\
\hline Age & & & Overall mean & \\
\hline Mean & 42 (SD 22) & 38 (SD 19) & 40 & 0.355 \\
\hline
\end{tabular}

The Chi-square and independent t-tests showed that sex and age were not significantly associated with ESBL phenotype Chi-square and independent t-test 


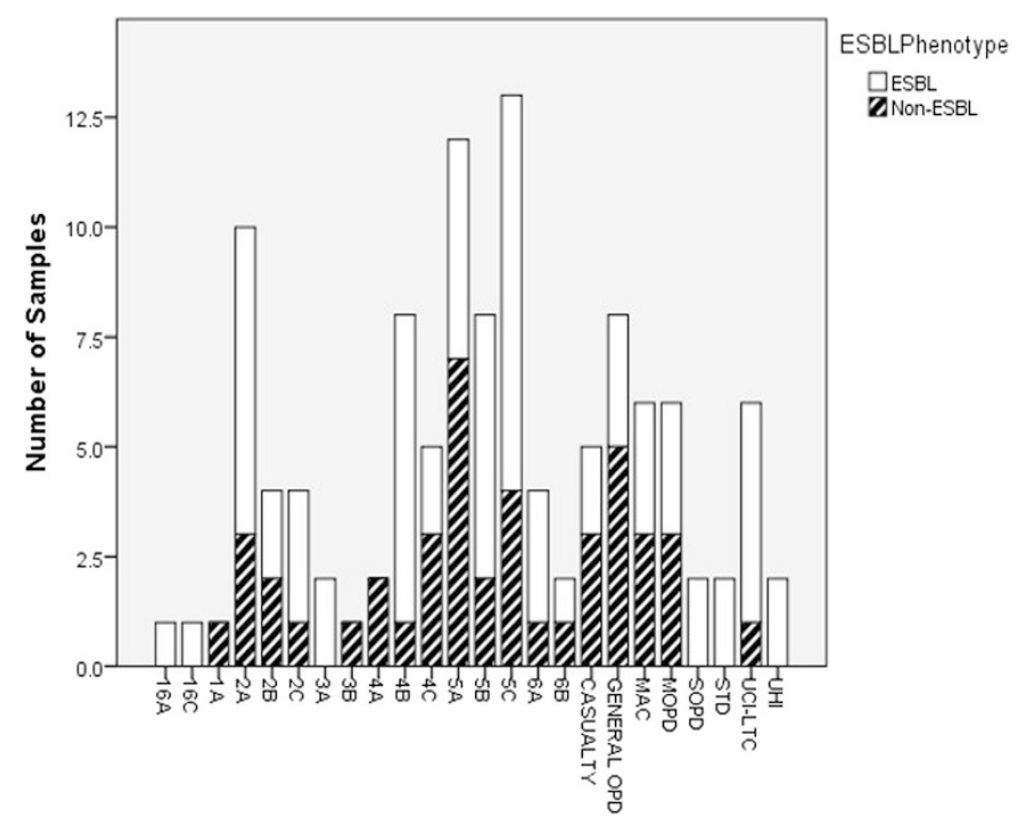

Ward

Fig. 1 Distribution of samples from the various wards. Surgical wards contributed the highest number of samples in the Microbiology laboratory during the study period. Surgical wards - 1A, 2A, 2B, 2C, 3A, 5A, 5B, 5C and SOPD; Medical wards - 3B, 4A, 4B, 4C, 6A, 6B, MOPD, MAC, UCI-LTC and UHI; Pediatric wards - 16A and 16C; General - OPD, MOPD, STD. Key: SOPD = Surgical Outpatients Dept; UCI-LTC = Uganda Cancer InstituteLiquid Tumor Cancer, MAC = Medical assessment Centre; MOPD = Medical outpatients Dept; OPD = Outpatients Dept; STD = Sexually Transmitted Diseases Dept; $\cup H I=$ Uganda Heart Institute

labelled and taken to the Microbiology Laboratory for Enterobacteriaceae culture and isolation. The originating ward, patient's gender and age were recorded. The samples were inoculated by streaking on Blood agar (Oxoid, UK), MacConkey agar (Oxoid, UK) and CLED agar (Oxoid, UK) plates. The plates were incubated aerobically at $37{ }^{\circ} \mathrm{C}$ for $18-24 \mathrm{~h}$ to allow development of bacterial colonies. Preliminary identification of the isolates was done using phenotypic colonial characteristics. Confirmatory identification of the suspect colonies was carried out by conventional biochemical tests as described by Cheesbrough [10]. These were: indole, Methyl red, Voges-Proskauer, citrate utilization and urease production tests as well as triple sugar iron and oxidase tests.

\section{Detection of ESBL-producing Enterobacteriaceae}

ESBL detection was based on the Double Disc Synergy Test and interpretation of the results done using the CLSI M100-S20 (2010) [11]. Briefly; 3-5 colonies of each isolate were picked from the growth plates with sterile wire loop, and suspended in $1 \mathrm{ml}$ of physiological saline. The resultant bacterial suspension was matched to the 0.5 McFarland turbidity standard so as to approximate the seeding density of the respective organisms. $100 \mu \mathrm{l}$ the bacterial suspension/broth culture were then surfacespread on Muller Hinton Agar (Oxoid, UK) plates using a sterile spreader. Antibiotic discs containing ceftazidime (CTC), cefotaxime (CTZ), ceftazidime-clavulanic acid (CFC) and cefotaxime-clavulanic acid (CTX) were placed on the plates which were incubated overnight at $37^{\circ} \mathrm{C}$. The zones of clearance $(\mathrm{mm})$ for the respective antibiotics were measured for each isolate using a divider and ruler. Organisms were considered to be ESBL-producers if the difference in zone of clearance between ceftazidime and ceftazidime-clavulanic acid or cefotaxime and cefotaximeclavulanic acid was $\geq 5 \mathrm{~mm}$. The prevalence of ESBLproducing bacteria was determined using the formula:

$$
\text { Prevalence }(\mathrm{P})=\frac{\text { Number of ESBL producing organisms }}{245} \times 100
$$

\section{Quality control}

Standard organisms (E. coli ATCC 25922 and K. pneumoniae ATCC 700603) were used to test for the potency of the antimicrobial discs (Table 1).

\section{Data analysis}

Clinical and socio-demographic data were entered into Epi Info ${ }^{\mathrm{TM}}$ v7 and exported to SPSSv21. Pearson Chisquare test was used to assess for any differences between the two ESBL phenotype categories with respect to clinical and demographic parameters. The means of the continuous variables, age and zones of clearance were 
Table 4 Association between ESBL phenotype and Enterobacteriaceae isolated, sample type and susceptibility pattern

\begin{tabular}{|c|c|c|c|}
\hline & ESBL (\%) & Non-ESBL (\%) & $P$ value \\
\hline \multicolumn{4}{|l|}{ Isolate } \\
\hline E. coli & $36(58.1)$ & $26(41.9)$ & 0.924 \\
\hline Klebsiella pneumoniae & $24(72.7)$ & $9(27.3)$ & 0.908 \\
\hline Enterobacter cloacae & $1(25)$ & $3(75)$ & 0.695 \\
\hline Proteus mirabilis & $10(62.5)$ & $6(37.5)$ & 0.999 \\
\hline \multicolumn{4}{|l|}{ Sample type } \\
\hline Urine & $46(63.9)$ & $26(36.1)$ & 0.980 \\
\hline HVS & $6(54.5)$ & $5(45.5)$ & 0.999 \\
\hline CSF & $1(100)$ & $0(0)$ & 0.999 \\
\hline Urethral swab & $1(100)$ & $0(0)$ & 1.000 \\
\hline Wound swab & $2(100)$ & $0(0)$ & 1.000 \\
\hline Blood & $2(40)$ & $3(60)$ & 1.000 \\
\hline Pus swab & $9(47.4)$ & $10(52.6)$ & 0.999 \\
\hline Surgical wound swab & $4(100)$ & $0(0)$ & 0.999 \\
\hline \multicolumn{4}{|l|}{ Ceftazidime Susceptibility } \\
\hline Resistant & $62(73.8)$ & $22(26.2)$ & 0.408 \\
\hline Intermediate & $4(36.4)$ & $7(63.6)$ & 0.187 \\
\hline Susceptible & $5(25)$ & $15(75)$ & 0.480 \\
\hline \multicolumn{4}{|l|}{ Cefotaxime Susceptibility } \\
\hline Resistant & $54(81.8)$ & $12(18.2)$ & 0.013 \\
\hline Intermediate & $4(30.8)$ & $9(69.2)$ & 0.067 \\
\hline Susceptible & $13(36.1)$ & $23(63.9)$ & 0.430 \\
\hline Binomial logistic regression & & & \\
\hline
\end{tabular}

When analysed using binomial logistic regression, ESBL phenotype was significantly associated with resistance to cefotaxime but not with resistance to ceftadizime, bacterial species or sample type compared using the Independent $t$-test. Crude logistic regression analysis was used to explore clinical and laboratory features of the ESBL phenotype for comparison with non-ESBL Enterobacteriaceae phenotypes. The differences were considered significant at $\mathrm{p}<0.05$.

\section{Ethical considerations}

The study protocol was approved by the Ethics Review Committee of the School of Biomedical Sciences of Makerere University Medical School. Permission was sought from the hospital and laboratory authorities. The ethical principles of scientific research as well as related national laws and regulations were strictly adhered to.

\section{Results}

The mean age of the participants was 40 years as shown in Table 2. Results indicated that 115 of the 245 samples (47 \%) had Enterobacteriaceae isolates. Of these isolates, $58.3 \%$ were from female patients while $41.7 \%$ were from males (Table 2). Statistical analysis of patient data using Chi-square and independent t-test indicated that gender and age were not significantly associated with ESBL phenotype. E. coli was the most isolated organism (53.9\%, n =62), followed by K. pneumoniae (28.7\%) as shown in Table 3. Most samples with Enterobacteriaceae isolates were from Obstetrics and Gynaecology wards i.e. 5A $(10.4 \%, \mathrm{n}=12)$ and $5 \mathrm{C}(11.3 \%, \mathrm{n}=13)$ (Fig. 1).

Results further showed that $62 \%$ of Enterobacteriaceae isolates were of the ESBL phenotype while $38 \%$ were of non-ESBL phenotype (Table 2). Most of the Enterobacteriaceae were isolated from the urine samples followed by pus samples as shown in Table 4 and Fig. 2 . However, just $64.9 \%$ and $47.4 \%$ of urine and pus isolates respectively were ESBL-producers. On the other hand,

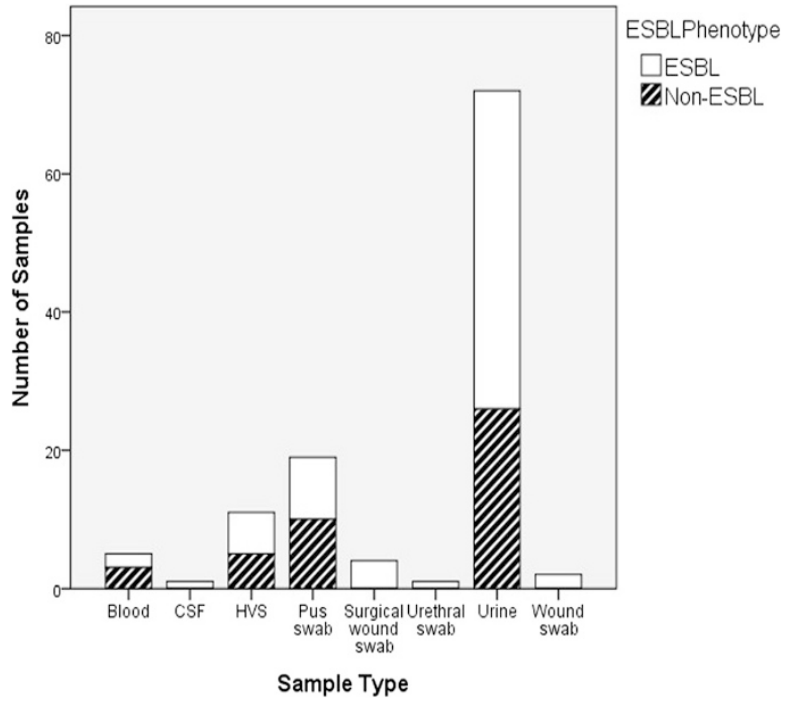

Fig. 2 Distribution of Enterobacteriaceae-positive samples. Most of the Enterobacteriaceae were isolated from urine samples 
Table 5 Mean zone of clearance $( \pm$ SD) of Enterobacteriaceae segregated by ESBL phenotype

\begin{tabular}{lllc}
\hline Antibiotic & ESBL & Non-ESBL & $P$ value \\
\hline Ceftazidime & $10.1 \pm 6.2$ & $18.1 \pm 7$ & 0.000 \\
Cefotaxime & $13.4 \pm 8.3$ & $21.6 \pm 8.3$ & 0.000 \\
Ceftazidime-Clavulanic acid & $22.3 \pm 6$ & $20.7 \pm 6.8$ & 0.198 \\
Cefotaxime-Clavulanic acid & $25.1 \pm 6.6$ & $22.3 \pm 8.3$ & 0.051 \\
\hline
\end{tabular}

The zones of clearance of ceftazidime and cefotaxime were statistically significantly associated with ESBL phenotype (Independent samples t-test)

albeit their small number, all isolates from CSF, wound and urethral swabs were ESBL producers (Fig. 2).

The mean zones of clearance for ESBL and non-ESBL phenotypes were lowest for ceftazidime $(10.1 \pm 6.2 \mathrm{~mm}$; $18.1 \pm 7 \mathrm{~mm}$ respectively) and highest for cefotaximeclavulanic acid ( $25.1 \pm 6.6 \mathrm{~mm} ; 22.3 \pm 8.3 \mathrm{~mm}$ respectively) as shown in Table 5 . The zones of clearance of ceftazidime and cefotaxime were significantly associated with ESBL phenotype ( $\mathrm{p}=0.000 ; 0.000$ respectively) while those of ceftazidime-clavulanic acid and cefotaxime-clavulanic acid were not ( $\mathrm{p}=0.198,0.051$ respectively).

There was a higher percentage of isolates showing resistance to ceftazidime (73\%) compared to cefotaxime (57.5 \%) as shown in Table 6. All Enterobacter cloacae isolates were resistant to both cefotaxime and ceftazidime. Table 4 shows that Klebsiella pneumoniae had the highest percentage of ESBL producers (72.7 \%). There were no statistically significant association between phenotype (ESBL/non-ESBL) and patients' age or gender (Table 4). Similarly, there were no significant association between phenotype (ESBL/non-ESBL) and species of Enterobacteriaceae (E. coli, Klebsiella pneumoniae, Enterobacter cloacae, Proteus mirabilis) or sample type (Table 4).

\section{Discussion}

The $3^{\text {rd }}$ and $4^{\text {th }}$ generation cephalosporins are often reserved for severe infections [12] but resistance to these drugs has been strikingly rapid worldwide [4]. Consequently, therapeutic options for the infections caused by the ESBL producers are becoming increasingly limited and; if available, expensive for low and middle income countries. The study revealed a slight female preponderance for ESBL-producing Enterobacteriaceae among the patients though gender was not statistically significant $(\mathrm{p}=0.089)$ as a factor. Kiratisin et al., [13] also revealed a female preponderance.

Urine samples constituted the greatest number of clinical samples in this study. According to Wilson and Gaido [14], urinary tract infections constitute the commonest bacterial infections and urine samples account for a significant percentage of samples in clinical microbiology laboratories worldwide. Most Enterobacteriaceae isolates were E. coli. Wilson and Gaido [14] indicates that E. coli is the most frequent cause of urinary tract infections and this could probably explain the high prevalence of $E$. coli isolates in our study. Similarly, studies in Tanzania [15] indicate that E. coli and Klebsiella pneumoniae are the most prevalent Enterobacteriaceae species in clinical samples. Furthermore, Maina et al., [16] in a study in Kenya reported higher prevalence for E. coli (53.8\%). Similar findings were reported in Bahrain [17].

In our study, the highest numbers of ESBL-producing isolates were from gynaecological and surgical wards. Many studies associate ESBL-producing Enterobacteriaceae with surgical wards. Studies by Seni et al., [9] show that most isolates from surgical wards are ESBL-producers. Prolonged hospital stay; inappropriate therapy; use of indwelling catheters, endotracheal/nasogastric tubes and severe illnesses are all possible drivers of their dissemination. Significantly, there is also movement of health workers between wards in the hospital and can migrate ESBLproducers from ward to ward leading to dissemination throughout the hospital.

The prevalence of ESBL producers among Enterobacteriaceae (62\%) was quite high compared to that reported by Moyo et al., [15] in Tanzania (45.2 \%). This wide variation in prevalence is probably due to differences in types of samples analysed and the extent of antibiotic use in the various wards. The present study reveals that $K$. pneumoniae and E. coli are major ESBL producers. Moyo et al., [15] showed $51.5 \%$ and $39.1 \%$ ESBL positivity among Klebsiella spp and E. coli respectively. On the other hand,

Table 6 Susceptibility pattern of Enterobacteriaceae to ceftazidime and cefotaxime

\begin{tabular}{|c|c|c|c|c|c|c|}
\hline & \multicolumn{3}{|c|}{ Ceftazidime (\%) } & \multicolumn{3}{|c|}{ Cefotaxime (\%) } \\
\hline & Resistant & Intermediate & Susceptible & Resistant & Intermediate & Susceptible \\
\hline E. coli & $41(66.1)$ & $7(11.3)$ & 14 (22.6) & 32 (51.6) & $9(14.5)$ & $21(33.9)$ \\
\hline E. cloacae & $4(100)$ & $0(0)$ & $0(0)$ & $3(75)$ & $0(0)$ & $1(25)$ \\
\hline K. pneumoniae & $27(81.8)$ & $1(3)$ & $5(15.2)$ & 21 (63.6) & $3(9.1)$ & $9(27.3)$ \\
\hline Proteus mirabilis & $12(75)$ & $3(18.8)$ & $1(6.3)$ & $10(62.5)$ & $1(6.3)$ & $5(31.2)$ \\
\hline$\%$ & 73.0 & 9.6 & 17.4 & 57.5 & 11.3 & 31.3 \\
\hline
\end{tabular}

There was a higher percentage of Enterobacteriaceae isolates showing resistance to ceftazidime than to cefotaxime. The interpretative criteria used was based on CLSI M100-S20 (2010) [11] where for ceftazidime (Resistant $\leq 17 \mathrm{~mm}$; Intermediate 18-20 mm; Susceptible $\geq 21 \mathrm{~mm}$ ) and for cefotaxime (Resistant $\leq 22 \mathrm{~mm}$; Intermediate 23-25 mm; Susceptible $\geq 26 \mathrm{~mm}$ ) 
Seni et al., [9] reported that $79.2 \%$ and $92.3 \%$ of E. coli and $K$. pneumoniae isolates are ESBL producers; further evidence that these two organisms account for most ESBL producers in the region. Our study showed a higher resistance to ceftazidime than to cefotaxime. On the other hand, Maina et al., [16] reported $21.2 \%$ resistance to ceftazidime and $65.4 \%$ resistance to cefotaxime. The differences seen in this study could be due to regional differences and the type of samples collected.

\section{Conclusions}

This study has demonstrated high prevalence of ESBLproducing Enterobacteriaceae in Mulago Hospital. The spread of these organisms reduces the antibiotic alternatives for the treatment of infections by these pathogens to mainly carbapenems; which are often reserved for lifethreatening infections. The study underscores the need for routine detection and reporting of ESBL-producers in Ugandan medical facilities so that measures are taken to avoid their uncontrolled spread and possible therapeutic failures. Clinicians need to be rational and judicious in use of antibiotics. An antibiotic use policy is also imperative to limit the dissemination of these organisms.

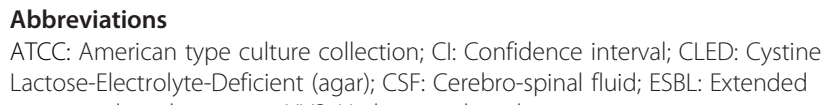

ATCC: American type culture collection; Cl: Confidence interval; CLED: Cystine Lactose-Electrolyte-Deficient (agar); CSF: Cerebro-spinal fluid; ESBL: Extended spectrum beta-lactamase; HVS: High vaginal swab.

\section{Competing interests}

The authors declare that they have no competing interests.

\section{Authors' contributions}

JNK, MLN and RK conceptualized the project, performed most of the lab experiments and wrote the manuscript. MLN contributed specific knowledge in conduction of the microbiological assays. CA assisted in statistically analysing the data. JGN assisted in finalizing the manuscript. All authors read and approved the final manuscript.

\section{Acknowledgements}

Special thanks go staff of Central Public Health Laboratory for their assistance during collection of the samples. We also like to acknowledge the Clinical Microbiology Laboratory of Mulago Hospital for their support in laboratory analysis of the samples. This study was financed by funds internally generated by the Pharmacology and Toxicology Research Lab of Makerere University.

Received: 23 December 2014 Accepted: 20 May 2015 Published online: 02 June 2015

\section{References}

1. Toth A, Juhasz-Kaszanyitzky E, Mag T, Hajbel-Vekony G, Paszti J, Damjanova I. Characterization of extended-spectrum beta-lactamase (ESBL) producing Escherichia coli strains isolated from animal and human clinical samples in Hungary in 2006-2007. Acta Microbiol Immunol Hung. 2013;60:175-85.

2. Hoban DJ, Lascols C, Nicolle LE, Badal R, Bouchillon S, Hackel M, et al. Antimicrobial susceptibility of Enterobacteriaceae, including molecular characterization of extended-spectrum beta-lactamase-producing species, in urinary tract isolates from hospitalized patients in North America and Europe: results from the SMART study 2009-2010. Diag Microbiol Infect Dis. 2012;74:62-7.

3. Akindele JA, Rotilu IO. Outbreak of neonatal Klebsiella septicaemia: a review of antimicrobial sensitivities. Afr J Med Med Sci. 1997;26:51-3.
4. Musoke RN, Revathi G. Emergence of multidrug-resistant gram-negative organisms in a neonatal unit and the therapeutic implications. J Trop Pediatr. 2000;46:86-91.

5. Dayan NH, Dabbah I, Weissman I, Aga I, Even L, Glikman D. Urinary Tract Infections Caused by Community-Acquired Extended-Spectrum beta-LactamaseProducing and Nonproducing Bacteria: A Comparative Study. J Pediatr. 2013;163:1417-21.

6. Badal RE, Bouchillon SK, Lob SH, Hackel MA, Hawser S, Hoban DJ. Etiology, Extended-Spectrum Beta-Lactamase Rates and Antimicrobial Susceptibility of Gram-negative Bacilli causing Intra-abdominal Infections in Patients in General Pediatric and Pediatric Intensive Care Units - Global Data from the Study for Monitoring Antimicrobial Resistance Trends 2008-2010. Pediatr Infect Dis J. 2013;32:636-40.

7. Agostinho A, Renzi G, Haustein T, Jourdan G, Bonfillon C, Rougemont M, et al. Epidemiology and acquisition of extended-spectrum beta-lactamaseproducing in a septic orthopedic ward. Springerplus. 2013;2:91.

8. Kish L. Sampling Organizations and Groups of Unequal Sizes. Am Sociol Rev. 1965;30:564-72.

9. Seni J, Najjuka CF, Kateete DP, Makobore P, Joloba ML, Kajumbula H, et al. Antimicrobial resistance in hospitalized surgical patients: a silently emerging public health concern in Uganda. BMC Research Notes. 2013;6:298.

10. Cheesebrough M: [District Laboratory Practice in Tropical Countries Part II]. Second Edition, Cambridge University Press 2006. p. 62. http://www. cambridge.org/us/academic/subjects/medicine/medicine-general-interest/ district-laboratorypractice-tropical-countries-part-2-2nd-edition.

11. Clinical and Laboratory Standards Institute. Performance Standards for Antimicrobial Susceptibility Testing: Twentieth Informational Supplement M100-S20. Wayne, PA, USA: CLSI; 2010

12. Trivedi M, Patel V, Soman R, Rodriguez C, Singhal T. The Outcome of Treating ESBL Infections with Carbapenems vs. Non Carbapenem Antimicrobials. J Assoc Physicians India. 2012;60:28-30.

13. Kiratisin $P$, Apisarnthanarak A, Laesripa C, Saifon P. Molecular characterization and epidemiology of extended spectrum-beta-lactamase-producing Escherichia coli and Klebsiella pneumoniae isolates causing health care associated infection in Thailand, where the CTX-M family is endemic. Antimicrob Agents Chemother. 2008;52:2818-24.

14. Wilson ML, Gaido L. Laboratory diagnosis of urinary tract infections in adult patients. Clinical Infectious Diseases. 2004;38:1150-8.

15. Moyo SJ, Aboud S, Kasubi M, Lyamuya EF, Maselle SY. Antimicrobial resistance among producers and non-producers of extended spectrum beta-lactamases in urinary isolates at a tertiary Hospital in Tanzania. BMC Research Notes. 2010;3:348.

16. Maina D, Revathi G, Kariuki S, Ozwara H. Genotypes and cephalosporin susceptibility in extended-spectrum $\beta$-lactamase producing enterobacteriaceae in the community. The Journal of Infection in Developing Countries. 2011;6:470-7.

17. Bindayna KM, Senok AC, Jamsheer AE. Prevalence of extended-spectrum beta-lactamase-producing Enterobacteriaceae in Bahrain. Journal of Infection and Public Health. 2009;2:129-35.

\section{Submit your next manuscript to BioMed Central and take full advantage of:}

- Convenient online submission

- Thorough peer review

- No space constraints or color figure charges

- Immediate publication on acceptance

- Inclusion in PubMed, CAS, Scopus and Google Scholar

- Research which is freely available for redistribution 